\title{
Development of a threshold positron spectrometer
}

\author{
M.C.A. Lopes ${ }^{\mathrm{a}, *}$, P.M. Gordo ${ }^{\mathrm{b}}$, A.P. de Lima ${ }^{\mathrm{b}}$, J.R. Mohallem ${ }^{\mathrm{c}}$ \\ ${ }^{a}$ Departamento de Fisica, Universidade Federal de Juiz de Fora, 36036-900 Juiz de Fora, MG, Brasil \\ ${ }^{\mathrm{b}}$ Departamento de Fisica, Universidade de Coimbra, Rua Larga, 3004-516 Coimbra, Portugal \\ ${ }^{\mathrm{c}}$ Departamento de Fisica, ICEx, Universidade Federal de Minas Gerais, P.O. Box 702, 30123-970 Belo Horizonte, Brasil
}

\begin{abstract}
We introduce a new positron spectroscopy experimental technique that we call threshold positron spectroscopy, by analogy with its similar threshold photoelectron spectroscopy. An apparatus that is being developed to explore this technique is described in this work.
\end{abstract}

(C) 2006 Elsevier Ltd. All rights reserved.

Keywords: Positron beam; Threshold positron spectrometer; Penetrating field technique

\section{Introduction}

Positron scattering by atoms and molecules is a special source of knowledge for both the positron-matter interaction and the physical properties of the target (Surko et al., 2005). This is because the selection rules are much more relaxed here when compared to those applied for example to photoionization or electron scattering experiments. At the target excitation by a positron, some special processes may take place as positronium (Ps) formation or pair-annihilation.

A promising new technique in the field would be the threshold positron spectroscopy (TPOS), projected in analogy with threshold photoelectron spectroscopy (TPES) (Lopes et al., 2006). TPOS would distinguish and detect very low $(\sim 20 \mathrm{meV})$ energy positrons scattered by a gaseous sample. Some possible advantages of TPOS over the traditional techniques could be advanced, as high sensitivity and high energy resolution (that however depend essentially on the incident positron beam energy resolution), which are known features of TPES. Furthermore, new kinds of resonances

\footnotetext{
*Corresponding author. Fax: + 553232293312.

E-mail address: clopes@fisica.ufjf.br (M.C.A. Lopes).
}

may appear and energy exchange due to post-collision interactions can be investigated.

Comparing to electron scattering, positron scattering experiments present some well known differences at low energies (Surko et al., 2005) that could be conveniently explored through the TPOS. Due to its characteristics of detecting nominally zero kinetic energy positrons, TPOS is potentially adequate to investigate the physics of the positron-atom attractive interactions either due to polarization of the target by the positron or to "chemical" formation of a Ps-ion complex.

In this paper, we present a project of an apparatus to perform TPOS, which is being assembled at the Physics Department of the Universidade Federal de Juiz de Fora, Brazil, covering the energy from 2 to $150 \mathrm{eV}$.

\section{Apparatus and experimental method}

The conception of TPO Spectrometer was essentially based on our threshold photoelectron spectrometer (Lopes et al., 2006), replacing however the photon beam by a positron tunable beam. The spectrometer consists of a tunable electrostatic positron beam, an effusive gas 
source and an analyzer based on the penetrating field technique (PFT) (Cvejanovic and Read, 1974), as shown in Fig. 1. The analyzer is tuned to accept only positrons of nominally zero kinetic energy and this tuning remains during all the experiment. The incident positron energy is scanned continually and may be tuned to the energies of highly excited states of target. As the positron energy is scanned across an excitation threshold, a close to zero energy positron is scattered, so that the TPO spectrum maps out the energy levels of the target. The positron beam is delivered to the spectrometer interaction region using a metal capillary of $3 \mathrm{~mm}$ internal diameter, isolating the beam chamber vacuum from other spectrometer elements. In order to prevent adverse charging effects acting on the efficiency collection of near zero energy charged particles, the capillary is electrically grounded. The collision region is also surrounded by a grounded cage. The spectrometer chamber is kept at a constant temperature of approximately $80^{\circ} \mathrm{C}$ during the experiment, thereby maintaining the stability of the spectrometer after the sample admission into the chamber. The positron beam and spectrometer chambers are recovered internally with a $0.05 \mathrm{~mm}$ layer of mu-metal to prevent spurious magnetic fields. The gas is introduced in the interaction chamber through a hypodermic needle of $0.7 \mathrm{~mm}$ internal diameter, situated approximately $3 \mathrm{~mm}$ from de positron beam and submitted to a small potential in order to improve the analyzer collection efficiency of nearly zero energy positrons. The requirement of the gas beam to perform TPOS is a beam density of approximately $10^{11}$ atoms $\mathrm{cm}^{-3}$ in an interaction region of $1 \mathrm{~mm}^{3}$ and a small angular spread (FWHM) less than $40^{\circ}$.

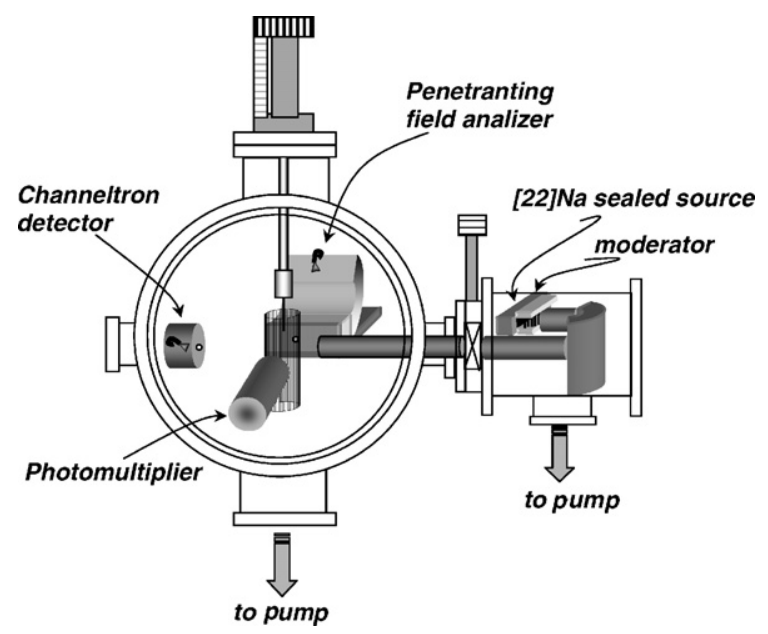

Fig. 1. Schematic diagram of the threshold positron spectrometer. The apparatus is installed inside a vacuum chamber internally covered by a mu-metal shield to reduce magnetic fields.
The scattered low-energy positrons extracted over $4 \pi$ sr are focalized and collimated at the entrance of a $127^{\circ}$ electrostatic deflector, using electrostatic lenses. The same analyzer can also be used to collect zero kinetic energy electrons, ejected from an ionized target, by changing the polarity of its electrostatic optics elements, as well as of the detector. The Ps formation is monitored during all the experiment by the energy analysis of the annihilation radiation detected by a NaI crystal coupled a photomultiplier. This detector will be substituted in the future, by a high-purity germanium detector (HPGe).

It is well known that the main difficult to perform positron scattering experiments is the low positron intensity, $10^{4}-10^{5} \mathrm{~s}^{-1}$ with energies spreads of a few electron volt from [22] Na source and W moderators. So, ideally the proposed experiment, TPOS, should be performed using positron facilities. However, the firsts steps on the development of TPO spectrometer will be done in our lab, using a $5 \mathrm{mCi}$ activity [22] $\mathrm{Na}$ source. This means that the first tests of the apparatus and first studies will be done using selected targets, focusing transitions with high cross sections. Our electrostatic positron beam using a $5 \mathrm{mCi}$ activity [22] $\mathrm{Na}$ source and a tungsten wire array moderator as "monoenergetic" positron generator, should provide an overall efficiency about $3.9 \times 10^{-4}$ (Liszkay et al., 2002). Considering the beam intensity of $1000 \mathrm{e}^{+} / \mathrm{mCi}$, it is expect to have about $5000 \mathrm{e}^{+} / \mathrm{s}$ emerging from moderator, with a pencil angle close to $15^{\circ}$ and an average energy of $2 \mathrm{eV}$ (Schultz and Lynn, 1988). Two electrostatic extractors electrodes placed few millimeters in front of the moderator, maximize the extraction of low-energy positrons emerging from the moderator and collect and focus them at the entrance of an electrostatic lens. This lens creates an image of this spot at the entrance of a deflector. A second set of electrostic lens, installed after the deflector, would efficiently accelerate and focalize the positron beam at the collision region. The intensity of the positron beam, expected to be about $4000 \mathrm{e}^{+} / \mathrm{s}$ at this region, can be monitored using single channel multiplier detector, sitting on the axis of the positron beam, approximately $20 \mathrm{~cm}$ from the interaction region. Considering the Helmholtz-Lagrange law, this lens contributes to reduce the spot size and beam collimation on target region. Once the energy resolution will be defined by the FWHM of the positron beam energy, it will be not crucial in this experiment the beam spot size on target and it can be bigger than the one usually applied on TPES. The large collection angle of TPOS does mean that any angular information is missed as all the threshold positrons are usually collected regardless of their initial ejection angle or even the impact angle. At the low energy regime, the magnitudes of the positronimpact scattering cross sections are, in many cases, larger than those for electrons (Surko et al., 2005). 
Moreover, at those conditions, when resonance phenomena are present, they produce sharp structures in the excitation function, enhancing the signal detected on TPO spectrum. Considering all these aspects and also the positron flux used on several positron cross-beam experiments published in the literature where angular dependence studies were performed and therefore the detection solid angle were much smaller (by instance $10^{5}$ $\mathrm{e}^{+} / \mathrm{s}$ by Detroit group (Hyder et al., 1986) and $\sim 6 \times 10^{3}$ $\mathrm{e}^{+} / \mathrm{s}$ by Bielefeld group (Falke et al., 1995)), it will be possible to performed TPOS applying the experimental condition of our apparatus. Surely, long time acquisition will be necessary to record the TPO spectrum.

\subsection{Threshold positron analyzer}

The threshold analyzer, working with the PFT (Cvejanovic and Read, 1974; King et al., 2001), employs two extractor elements placed in front of its entrance aperture, which produce a weak electrostatic field in the interaction region. This field produces a potential well that preferentially extracts and collects zero energy scattered positrons over $4 \pi \mathrm{sr}$. A set of two electrostatic lenses accelerates the positrons up to $2 \mathrm{eV}$ and focuses them to the entrance of a $127^{\circ}$ cylindrical dispersive selector, which is employed to discriminate high-energy positrons scattered into the solid angle defined by the extracting electrode apertures. In fact, the high-energy resolution of this analyzer is obtained by virtue of the very fast fall of in the collection efficiency with energy; simultaneously, a very large solid angle of collection $(4 \pi \mathrm{sr})$ provides the high efficiency of the system.

The analyzer was built at our laboratory at Universidade Federal de Juiz de Fora, and was extensively tested by means of argon and $\mathrm{N}_{2}$ threshold photoelectron spectra, recorded using synchrotron radiation at the Brazilian Light Source (Laboratório Nacional de Luz Síncrotron, LNLS). An excellent performance was attained. We have obtained spectra with a resolution of $17 \mathrm{meV}$ at $12 \mathrm{eV}$, that amounts a resolving power $(E / \Delta E)$ above 700 , with a high signal/noise ratio. In Fig. 2 is shown the resolution obtained in the argon TPE spectrum and in Fig. 3 the TPE spectrum of $\mathrm{N}_{2}$.

\section{Conclusions}

In order to gain information on the possible mechanism involved on the positron-atom and -molecule collisions, we are assembling a threshold positron spectrometer (TPOS) covering the impact energy range from 2 to $150 \mathrm{eV}$. TPOS can constitute an important advance in this investigation, due to its important characteristics of high sensitivity and high-energy resolution. A comparison between positron and electron excitation cross sections between atomic or molecular

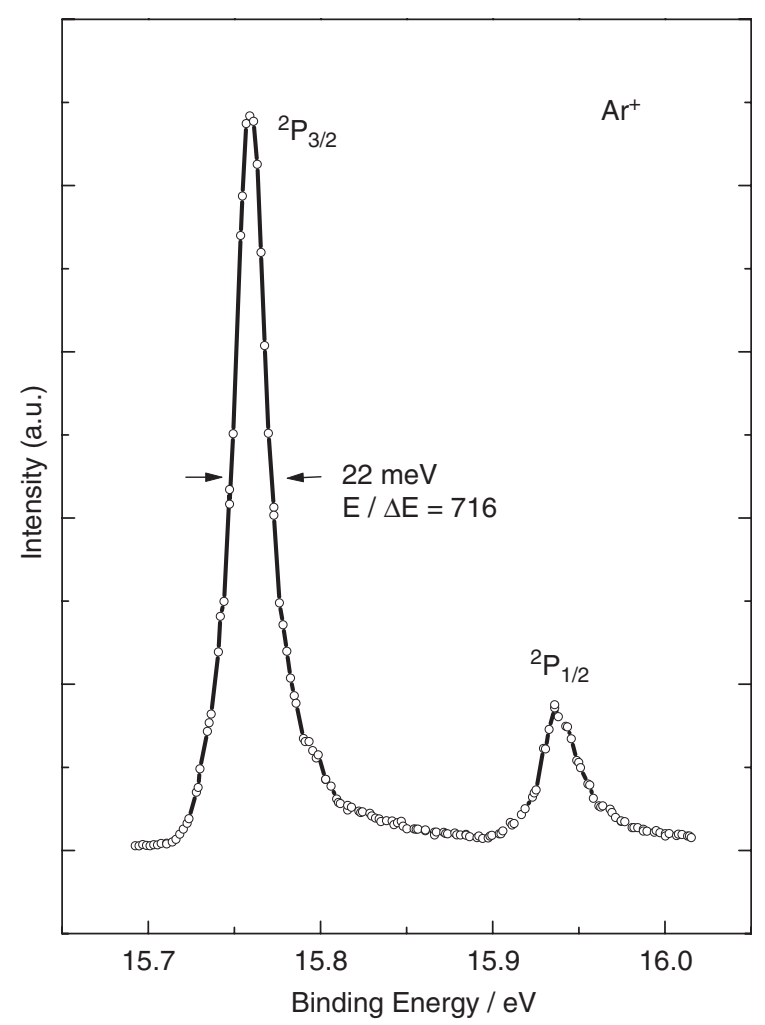

Fig. 2. Threshold photoelectron spectrum of argon, recorded with an energy resolution of $17 \mathrm{meV}$ at $12 \mathrm{eV}$, that corresponds to a resolving power $(E / \Delta E)$ better than 700 .

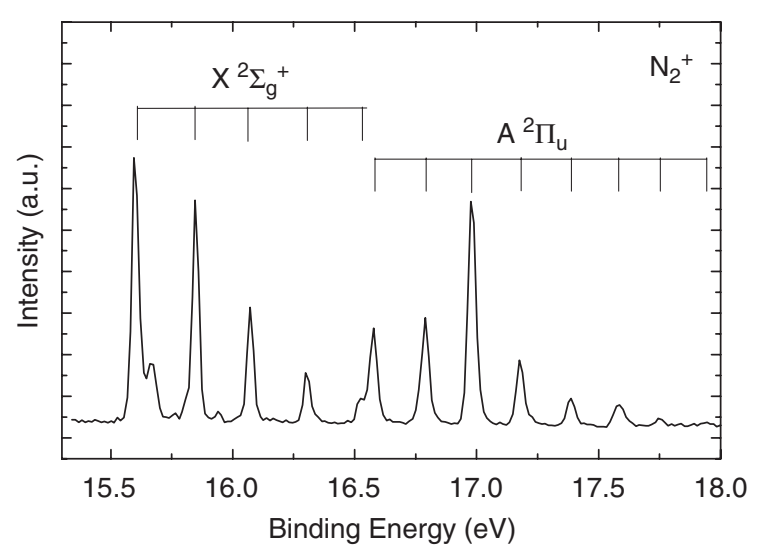

Fig. 3. Threshold photoelectron spectrum of $\mathrm{N}_{2}$ acquired in the $15.2-18.0 \mathrm{eV}$ energy range, with $5 \mathrm{meV}$ steps. The count rate at the peak related to the $v=0, X^{2} \Sigma_{\mathrm{g}}^{+}$ionic state is $7.0 \mathrm{kHz}$.

energy states can indeed provide new and important information on the electronic states involved. This is the first technique through which this results can become available. 


\section{Acknowledgments}

We are grateful for financial support from $\mathrm{CNPq}$, CAPES, FAPEMIG (Brazilian agencies) and LNLS.

\section{References}

Cvejanovic, S., Read, F.H., 1974. New technique for threshold excitation spectroscopy. J. Phys. B: At. Mol. Opt. Phys. 7 (10), 1180-1193.

Falke, T., Raith., W., Weber, M., Wesskamp, U., 1995. Differential positronium formation in positron-argon collisions. J. Phys. B: At. Mol. Opt. Phys. 28, L505-L509.

Hyder, G.M.A., Dababneh, M.S., Hseih, Y.-F., Kauppila, N.E., Kwan, C.K., Mahdavi-Hezadeh, M., Stein, T.S., 1986. Positron differential elastic-scattering cross-section measurements for argon. Phys. Rev. Lett. 57 (18), 2252-2255.

King, G.C., Yencha, A.J., Lopes, M.C.A., 2001. Threshold photoelectron spectroscopy using synchrotron radiation. J. Electron Spectrosc. Rel. Phen. 114, 33-40.

Liszkay, L., Kajcsos, Zs., Barthe, M.-F., Henry, L., Duplatre, G., Nagy, A., 2002. Improved tungsten moderator structures for slow positron beams. Appl. Surf. Sci. 194, 16-19.

Lopes, M.C.A., Couto, H., Moreira, C.D., Silva, H., 2006. Atomic and molecular threshold photoelectron spectroscopy. Quimica Nova 29 (1), 156-159, and references therein.

Schultz, P.J., Lynn, K.G., 1988. Interaction of positron beams with surfaces, thin-films, and interfaces. Rev. Mod. Phys. 60 (3), 701-779.

Surko, C.M., Gripakin, G.F., Buckman, S.J., 2005. Low energy positron interactions with atoms and molecules. J. Phys. B: At. Mol. Opt. Phys. 38 (6), R57-R126. 